\title{
Influence of Admission to a Tertiary Care Hospital after a Fall on Use of Potentially Inappropriate Medications among Older Patients
}

\author{
Erin Francis, Derek Dyks, and Salmaan Kanji
}

\begin{abstract}
Background: Each year, about one-third of individuals over the age of 65 years will experience a fall, and half of these will experience a subsequent fall in the following year. The use of potentially inappropriate medications (PIMs) is an important factor contributing to increased fall risk in geriatric patients.

Objective: To determine the proportion of patients over the age of 65 admitted to orthopedics and general medicine services with diagnosis of a fall who experienced a change in the total number or dosage of PIMs, as defined by the Beers criteria, upon discharge from hospital.

Methods: This retrospective observational study involved patients admitted to a tertiary care hospital with diagnosis of a fall between January 1 and December 31, 2011. Those aged 65 years or older with at least one PIM on admission were eligible for inclusion. Data analysis included $\chi^{2}$ and Fisher testing, as well as multivariate analysis.

Results: A total of 148 patients were included, of whom 63 (43\%) had an overall change in the dosage or number of PIMs during their hospital stay. Forty patients $(27 \%)$ had an overall reduction in the dosage or number of PIMs upon discharge from hospital, whereas 23 (16\%) experienced an overall increase in the dosage or total number of PIMs. The mean number ( \pm standard deviation) of PIMs decreased during the hospital stay, from $1.6 \pm 0.8$ on admission to $1.4 \pm 0.9$ on discharge $(p=0.03)$. Benzodiazepines were the class of PIMs most frequently discontinued or reduced in dosage.

Conclusion: One-quarter of patients admitted with falls had de-escalation of PIMs upon hospital discharge. Although dosage reduction or drug discontinuation may not be appropriate for all patients, a standardized approach to medication review during the hospital stay and improved prescriber education and awareness of PIM use among elderly individuals are warranted.
\end{abstract}

Keywords: falls, potentially inappropriate medications, older adults, hospital admission

Can J Hosp Pharm. 2014;67(6):429-35

\section{RÉSUMÉ}

Contexte : Chaque année, environ le tiers des personnes de plus de 65 ans subiront une chute et la moitié d'entre elles chuteront de nouveau au cours de l'année suivante. Or, l'utilisation de médicaments potentiellement inappropriés (MPI) représente un facteur important d'augmentation des risques de chute chez le patient âgé.

Objectif : Déterminer la proportion des patients de plus de 65 ans admis aux services de médecine générale et d'orthopédie en raison d'un diagnostic de chute pour qui le nombre ou la posologie de MPI, selon les critères de Beers, avaient changé au moment du congé.

Méthodes : Cette étude d'observation rétrospective a porté sur des patients ayant subi une chute et ayant été hospitalisés dans un centre hospitalier de soins tertiaires entre le ler janvier et le 31 décembre 2011. Parmi ceux-ci, seuls les patients âgés de 65 ans ou plus chez qui l'on a noté au moins un MPI au moment de l'hospitalisation étaient admissibles. L'analyse des données reposait sur le test du $\chi^{2}$, le test de Fisher ainsi que sur une analyse multivariée.

Résultats : En tout, 148 patients ont été retenus. Parmi eux, 63 (43\%) ont connu un changement à la posologie de MPI ou au nombre de ces derniers au cours de leur séjour à l'hôpital. On a noté au moment du congé que 40 patients $(27 \%)$ avaient fait l'objet d'une réduction globale de la posologie de leurs MPI ou du nombre de ceux-ci et que 23 patients $(16 \%)$ avaient connu une augmentation globale de la posologie de leurs MPI ou du nombre de ceux-ci. Le nombre moyen ( \pm l'écart-type) de MPI a diminué au cours du séjour à l'hôpital, passant de 1,6 $\pm 0,8$ au moment de l'hospitalisation à $1,4 \pm 0,9$ au moment du congé $(p=0,03)$. Les benzodiazépines représentaient la classe de MPI qui était la plus fréquemment interrompue ou dont la posologie était la plus souvent réduite.

Conclusions : Le quart des patients hospitalisés pour une chute avait fait l'objet d'un allégement de MPI au moment du congé. Bien qu'une réduction de la posologie ou l'interruption de la prise d'un médicament ne soient pas nécessairement recommandées pour tous les patients, il est justifié d'adopter une approche standardisée de l'évaluation de la médication pendant le séjour à l'hôpital, et de mieux instruire et sensibiliser les prescripteurs sur les enjeux liés aux MPI chez les personnes âgées.

Mots clés : chutes, médicaments potentiellement inappropriés, personnes âgées, hospitalisation

[Treduction par l'éditeur] 


\section{INTRODUCTION}

$\mathrm{M}$

edical and therapeutic advances have contributed to an increase in life expectancy by slowing the progression and delaying the consequences of chronic illness. ${ }^{1,2}$ Older adults represent the most rapidly growing segment of the global population, a shift that is placing increased pressure on the health care system. ${ }^{3}$ Many elderly people suffer from multiple chronic diseases and become major consumers of medication. ${ }^{1}$ Polypharmacy, of which one accepted definition is the regular use of 4 or more medications, ${ }^{3}$ is prevalent among elderly patients and can be the cause of iatrogenic complications if not managed appropriately. ${ }^{3}$

For many drugs, the pharmacological action may differ between geriatric and younger individuals. This difference may be the result of physiologic changes related to the aging process or to age-related decline in organ function. Such changes can affect the pharmacokinetics or pharmacodynamics of medications. The effects of aging can manifest as an increase or decrease in the intended pharmacological action of a medication, which in turn increases the risk of adverse drug events (ADEs). ${ }^{2}$ Compared with younger individuals, patients over the age of 65 years are at higher risk of experiencing an ADE. ${ }^{4}$ ADEs, such as falls, can lead to a decline in functional status of older adults or admission to hospital and may influence their ability to live independently.

Each year, about one-third of individuals over the age of 65 years living in the community will experience a fall, ,5 and about half of these will have a subsequent fall in the following year. ${ }^{6,7}$ Fall-related complications such as fracture, death, admission to hospital, admission to an institution, and disability increase with age. ${ }^{8}$ The risk factors for falls among elderly people are often multifactorial and may be intrinsic (e.g., muscle weakness, impaired balance), environmental (e.g., slippery surfaces), or extrinsic (e.g., medications, polypharmacy). ${ }^{?}$

A medication can increase fall risk through a combination of mechanisms, such as excessive sedation, impaired balance, cognitive impairment, and orthostatic reactions. ${ }^{10}$ When treating elderly patients, it is important to consider both the intended beneficial effect of treatment and the acceptable level of risk for ADEs, such as falls, as a result of drug therapy.

Observational studies have suggested that the use of potentially inappropriate medications (PIMs) can increase fall risk among geriatric patients. ${ }^{5}$ A PIM is defined as a medication for which the potential risk for ADEs outweighs the possible clinical benefit for the patient. ${ }^{11,12}$ The well-known Beers criteria define PIMs with the potential to increase fall risk in elderly individuals, and it is recommended that certain medication classes be avoided for elderly patients with a history of falls (unless safer alternatives are not available). ${ }^{12}$ We believe that admission to hospital provides an opportunity to make changes in the type of drugs and the total number of PIMs prescribed. ${ }^{1}$

The goal of this study was to determine the influence of hospital admission on the use of medications known to increase the risk of falls in older adults (as defined by the 2012 Beers criteria) among patients admitted to the authors' tertiary care facility after experiencing a fall. The primary objective was to determine the proportion of patients over the age of 65 years who were admitted to hospital with a diagnosis of falls and at least one PIM identified on a best possible medication history $(\mathrm{BPMH})$ at the time of admission who had a change in the total number or dosage of PIMs upon discharge. The secondary objective was to compare the frequency of subsequent falls between patients discharged with no change, an increase, or a decrease in the total number or dosage of PIMs. Specific insights were sought into prescribers' awareness of PIMs in elderly patients and whether appropriate medication changes were being made to reduce the risk of subsequent falls in patients who were using PIMs at the time of hospital admission.

\section{METHODS}

\section{Patient Selection}

For this retrospective observational study, the study population was drawn from a coded list generated by the medical records department at The Ottawa Hospital (an 1150-bed institution) identifying patients 65 years of age or older admitted to the orthopedics or general medicine service with diagnosis of a fall between January 1 and December 31, 2011. Patients were eligible for inclusion if they met the following criteria: age 65 years or older, medication history completed on hospital admission (as part of the institution's medication reconciliation program), taking one or more PIMs defined by the Beers criteria as inappropriate for those who have experienced a fall or a fracture (as documented on the admission medication history), and admission to hospital with diagnosis of a fall (based on codes for falls from the International Classification of Diseases and Related Health Problems, 10th revision, as modified for Canada [ICD-10-CA]: codes W00-W19). For the purpose of this study, PIMs (as per the Beers Criteria) were anticonvulsants (unless a documented seizure disorder was recorded), antipsychotics, benzodiazepines, non-benzodiazepine hypnotics (zopiclone, zolpidem), selective serotonin reuptake inhibitors (SSRIs), and tricyclic antidepressants (TCAs). ${ }^{12}$ Patients who died during the index hospital stay were excluded.

\section{Data Collection}

During the study period, 756 patients 65 years of age or older were admitted to the orthopedic and general medicine services at the authors' institution with diagnosis of a fall. A convenience sample size of 150 patients was chosen based on an estimated subsequent fall rate of 50\%., ${ }^{6,7}$ The sampling strategy was created by dividing the total number of patients admitted (756) by the sample size (150) to determine a sequence for patient enrolment. On this basis, every fifth patient on a chronological list of admissions was screened for enrolment, to avoid bias related to seasonal variation in fall risk and incident density. 
If a patient selected in this way did not meet the inclusion criteria, charts before and after the selected chart on the admission list were alternately screened until a patient meeting the inclusion criteria was identified.

The following data were retrieved from the electronic medical records of patients meeting the inclusion criteria: age, sex, admitting service for the index fall, medications and corresponding dosages used before hospital admission (as listed on the BPMH), and medications and corresponding dosages prescribed on hospital discharge (as shown on electronic discharge prescriptions, discharge summaries, medication administration records, or handwritten physician orders). The total number and dosage of PIMs continued on discharge were compared with the admission BPMH to determine whether there had been a reduction, an increase, or no change in the total number or dosage of PIMs during the hospital stay. Consultations to the geriatrics service and pharmacist involvement in the patient's care (defined as any written documentation of an activity related to patient care in the medical chart) were also recorded, to determine the influence of these factors on the primary outcome.

To determine whether study patients experienced a subsequent fall after the index event, coded lists generated by the medical records department were screened for a diagnosis of falls that resulted in presentation to the emergency department or readmission to the institution over the following year (i.e., January 1 to December 31, 2012).

\section{Statistical Analysis}

The proportion of patients for whom there was a reduction, an increase, or no change in the total number or dosage of PIMs on hospital discharge, relative to the admission BPMH, was calculated and described according to targeted medication class.
The mean numbers of PIMs among individuals on hospital admission and discharge were compared with a paired $t$ test. The frequency of subsequent falls was calculated as a proportion. The proportion of patients with falls who had a reduction in the total number or dosage of PIMs on discharge was compared with the proportion who had either an increase or no change in the total number or dosage of PIMs with the Fisher exact test.

The proportion of patients who experienced drug discontinuation or a dosage reduction was compared with the proportion of patients who did not experience drug discontinuation or a dosage reduction using the following covariates (identified a priori): admitting service, presence or absence of a geriatrics consultation, and presence or absence of pharmacist involvement in patient care. These data were analyzed with the Fisher exact test or $\chi^{2}$ analysis to determine the influence of these factors on reduction in total number or dosage of PIMs on discharge.

To examine predictors of dosage reductions or discontinuation of targeted drugs, logistic regression analysis was conducted in which dosage reduction or discontinuation of targeted drugs was the dependent variable. Four predetermined factors (geriatrics consult, pharmacist involvement, age, and admitting service) were introduced into the model simultaneously. The results are presented as odds ratios (ORs) with 95\% confidence intervals (CIs).

All statistical analyses were performed with SPSS software (IBM, Armonk, New York); $p$ values less than 0.05 were considered statistically significant.

\section{RESULTS}

Electronic medical records for 373 patients were screened, and 148 patients met the inclusion criteria for study enrolment (Figure 1). The study population consisted of 119 women (80\%)

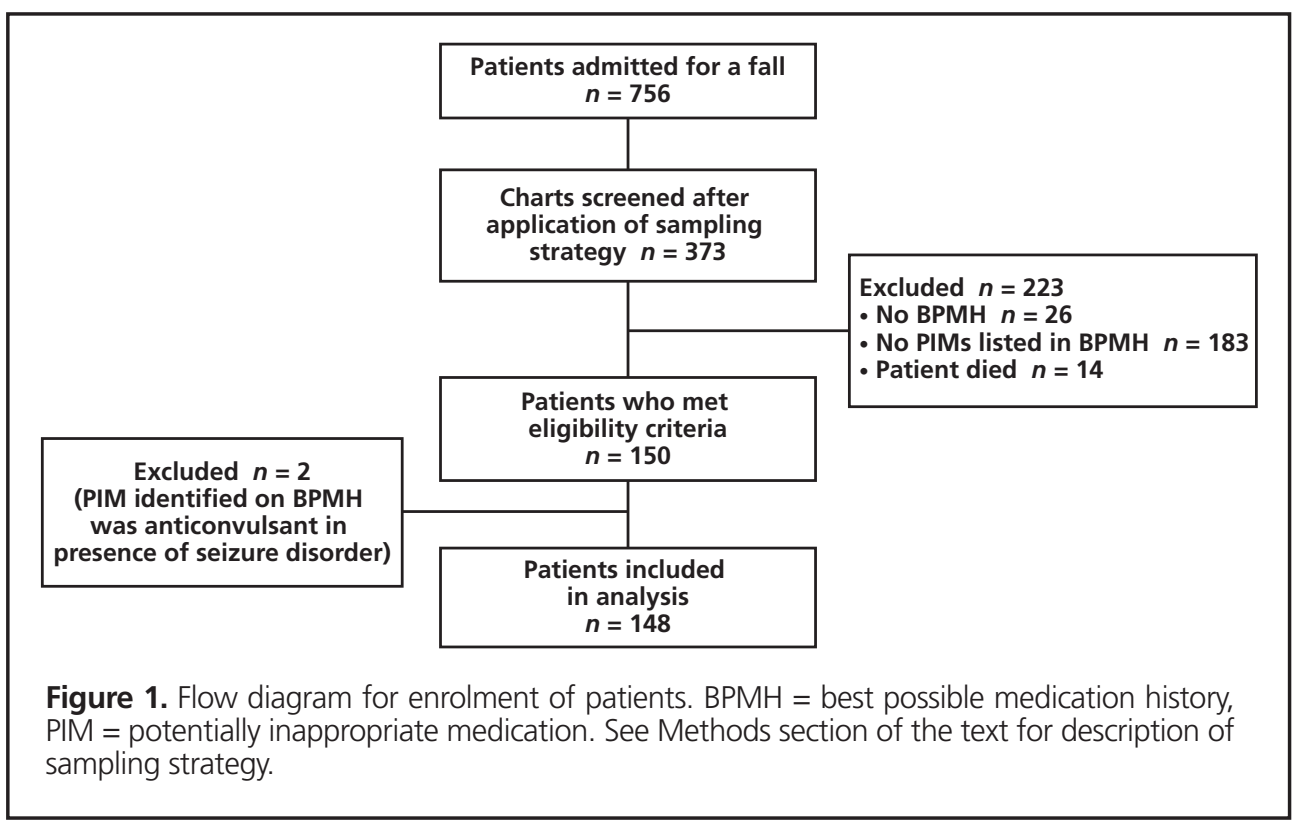


and 29 men (20\%), with mean age \pm standard deviation of $82 \pm$ 8.2 years (range $65-102$ years). Of the 148 patients in the study population, $121(82 \%)$ were admitted to the orthopedics service and $27(18 \%)$ to the general medicine service. On admission to hospital, the mean number of PIMs per patient was $1.6 \pm 0.8$, and all patients had at least one targeted PIM on their admission medication history. The most frequently prescribed PIMs at the time of admission were SSRIs (68 patients [46\%]) and benzodiazepines (66 patients [45\%]) (Table 1).

Of the 148 patients, $63(43 \%)$ had a change in the total number or dosage of PIMs during the hospital stay. Forty patients (27\%) experienced a dosage reduction or discontinuation of a PIM during the hospital stay $(p<0.001)$. Twenty-three patients $(16 \%)$ had a greater number or an increased dosage of PIMs at discharge. The mean number of PIMs declined to $1.4 \pm 0.9$ on hospital discharge $(p=0.03)$.

During the follow-up period, 13 (9\%) of the patients experienced a subsequent fall that resulted in presentation to the emergency department or readmission to hospital. Among the 40 patients discharged from hospital with fewer PIMs or a reduction in PIM dosage, 2 (5\%) experienced a second fall, compared with $11(10 \%)$ of the 108 patients without a reduction in number or dosage of PIMs $(p=0.52)$ (Table 2). Recurrent falls occurred in $2(9 \%)$ of the 23 patients discharged from hospital with a greater number or increased dosage of PIMs and $11(9 \%)$ of the 125 patients discharged on the same number or fewer PIMs $(p>0.99)$.

Benzodiazepines constituted the PIM class with the highest rates of discontinuation and dosage reduction (Table 3 ). On admission, 66 patients had a prescription for at least one benzodiazepine, with 17 (26\%) experiencing discontinuation and $9(14 \%)$ experiencing a dosage reduction during the hospital stay. Of the 68 patients who were taking an SSRI on admission, only $5(7 \%)$ experienced discontinuation and only 1 (1\%) experienced dosage reduction while in the hospital. Antipsychotics constituted the most frequently added medication class on hospital discharge (with 13 [9\%] of enrolled patients having a new antipsychotic prescribed at the time of discharge).

Multivariate analysis suggested that a geriatrics consultation was a predictor of discontinuation or dosage reduction of PIMs on hospital discharge (OR 6.7, 95\% CI 2.8-16.5; $p<0.001$ ). Admission to the orthopedics service was also a predictor of discontinuation or dosage reduction of PIMs on hospital discharge (OR 2.7, 95\% CI 1.0-7.4; $p=0.044$ ). Pharmacist involvement in patient care during the hospital stay (OR 1.8, 95\% CI 0.6-5.0, $p=0.27$ ) and patient age (OR 1.04, 95\% CI

\section{Table 1. Potentially Inappropriate Medications (PIMs) on Admission and Discharge}

\begin{tabular}{lrrrr} 
& \multicolumn{3}{c}{ No. (\%) of Patients* $(\boldsymbol{n}=\mathbf{1 4 8})$} \\
\cline { 2 - 6 } Medication Class & \multicolumn{2}{c}{$\begin{array}{c}\text { Present on } \\
\text { Admission }\end{array}$} & $\begin{array}{c}\text { Present on } \\
\text { Discharge }\end{array}$ \\
\hline Anticonvulsant & 24 & $(16)$ & 26 & $(18)$ \\
\hline Antipsychotic & 38 & $(26)$ & 45 & $(30)$ \\
\hline Benzodiazepine & 66 & $(45)$ & 52 & $(35)$ \\
\hline Non-benzodiazepine hypnotic & 13 & $(9)$ & 14 & $(9)$ \\
\hline Tricyclic antidepressant & 13 & $(9)$ & 12 & $(8)$ \\
\hline Selective serotonin reuptake inhibitor & 68 & $(46)$ & 63 & $(43)$ \\
\hline Potentially inappropriate medications & & & & \\
0 & 0 & $(0)$ & 12 & $(8)$ \\
$\geq 1$ & 148 & $(100)$ & 136 & $(92)$ \\
$\geq 2$ & 88 & $(59)$ & 62 & $(42)$ \\
$\geq 3$ & 22 & $(15)$ & 18 & $(12)$ \\
$\geq 4$ & 4 & $(3)$ & 6 & $(4)$ \\
5 & 1 & $(1)$ & 0 & $(0)$ \\
\hline
\end{tabular}

* Percentages for the 6 medication classes do not sum to $100 \%$ because some patients were taking multiple PIMs from 2 or more medication classes.

Table 2. Univariate Comparison of Patients with and without De-escalation of Potentially Inappropriate Medications

\begin{tabular}{lccccc} 
Variable & $\begin{array}{c}\text { Dosage Decrease or } \\
\text { Drug Discontinued } \\
(\boldsymbol{n}=\mathbf{4 0 )}\end{array}$ & $\begin{array}{c}\text { No Dosage Decrease, } \\
\text { Drug Not Discontinued } \\
(\boldsymbol{n}=\mathbf{1 0 8})\end{array}$ & $\boldsymbol{p}$ Value \\
\hline Geriatrics consultation & 18 & $(45)$ & 13 & $(12)$ & $<0.001$ \\
Pharmacist involvement & 10 & $(25)$ & 13 & $(12)$ & 0.07 \\
Recurrent falls & 2 & $(5)$ & 11 & $(10)$ & 0.52 \\
Admitted to orthopedics & 29 & $(73)$ & 92 & $(85)$ & 0.08 \\
\hline
\end{tabular}


This single copy is for your personal, non-commercial use only.

For permission to reprint multiple copies or to order presentation-ready copies for distribution, contact CJHP at cjhpedit@cshp.ca

Table 3. Changes to Prescriptions for Potentially Inappropriate Medications (PIMs) During Hospital Stay

\begin{tabular}{|c|c|c|c|c|c|c|}
\hline Medication Class & $\begin{array}{c}\text { No. of } \\
\text { Prescriptions } \\
\text { on Admission }\end{array}$ & $\begin{array}{l}\text { No. } \\
\text { Pres } \\
\text { with } \\
\text { Inc }\end{array}$ & $\begin{array}{l}\% \text { ) of } \\
\text { iptions } \\
\text { Dosage } \\
\text { ease* }\end{array}$ & $\begin{array}{c}\text { No. }(\%) \text { of } \\
\text { Prescriptions } \\
\text { with Dosage } \\
\text { Decrease* }\end{array}$ & $\begin{array}{c}\text { No. of } \\
\text { Prescriptions } \\
\text { for New Drug }\end{array}$ & $\begin{array}{c}\text { No. of } \\
\text { Prescriptions } \\
\text { Discontinued }\end{array}$ \\
\hline Anticonvulsants & 28 & 1 & (4) & $2 \quad(7)$ & 3 & 0 \\
\hline Antipsychotics & 43 & 2 & (5) & $5 \quad(12)$ & 13 & 6 \\
\hline Benzodiazepines & 71 & 5 & (7) & 9 (13) & 0 & 17 \\
\hline $\begin{array}{l}\text { Non-benzodiazepine } \\
\text { hypnotics }\end{array}$ & 13 & 1 & (8) & (0) & 2 & 1 \\
\hline Tricyclic antidepressants & 13 & 0 & (0) & (0) & 1 & 2 \\
\hline $\begin{array}{l}\text { Selective serotonin } \\
\text { reuptake inhibitors }\end{array}$ & 68 & 2 & (3) & (1) & 0 & 5 \\
\hline All PIMs & 236 & 11 & (5) & 17 & 19 & 31 \\
\hline
\end{tabular}

*Percentages are calculated from the total number of prescriptions at baseline, as shown in column 2 of the table. For 3 medication classes (anticonvulsants, antipsychotics, and benzodiazepines), the total number of prescriptions was greater than the number of patients receiving that class of medication (as shown in Table 1), because some patients were taking more than one drug from the medication class.

$0.94-1.0, p=0.61)$ were not predictors of discontinuation or dosage reduction of PIMs on hospital discharge.

\section{DISCUSSION}

In this study, admission to hospital after a fall resulted in discontinuation or dosage reduction of PIMs in 40 (27\%) of the enrolled patients. An additional 23 patients (16\%) had a new PIM added to their regimen or an increase in dosage of an existing PIM, such that a total of 63 patients $(43 \%)$ had a change in the total number or dosage of PIMs during the hospital stay. Multivariate analysis showed that a consultation with the geriatrics service, in combination with admission to the orthopedics service, was a predictor of discontinuation or dosage reduction of PIMs.

Benzodiazepines and SSRIs were the most frequently prescribed PIMs at the time of hospital admission, and benzodiazepines were the PIMs most frequently discontinued or with dosage reductions on discharge from hospital. According to the literature, benzodiazepines are the most commonly prescribed psychotropic medication and are known to be associated with falls. ${ }^{13}$ For example, in their meta-analysis, Leipzig and others ${ }^{14}$ reported an adjusted OR of 1.48 (95\% CI 1.23-1.77) for falls among users of benzodiazepines.

Several studies investigating use of PIMs by geriatric patients have been based on the criteria for determining inappropriate medication use developed by Beers and others. ${ }^{1}$ The original criteria were developed in 1991 using a modified Delphi method to create a list of PIMs for nursing home residents. The criteria were revised and expanded in 1997 and again in 2003 to encompass all geriatric care settings. These criteria, which represent a widely cited tool to identify potentially harmful medications in elderly patients, were most recently updated in $2012 .{ }^{12}$ The Beers criteria identify medications that are viewed as having an unfavourable balance of risks and benefits for older adults. The 53 medication classes in the updated criteria are categorized as follows: (1) potentially inappropriate medications and classes to be avoided in older adults, (2) potentially inappropriate medications and classes to be avoided in older adults who have certain diseases and syndromes that could be exacerbated by the listed drugs, and (3) medications to be used with caution in older adults. ${ }^{12}$ The current study focused on medications in the first of these categories, which should be avoided in patients with a history of falls or fractures (unless safer alternatives are not available). On the basis of high-quality evidence, the expert panel responsible for the criteria strongly recommend avoiding anticonvulsants (unless a seizure disorder is present), antipsychotics, benzodiazepines, non-benzodiazepine hypnotics, TCAs, and SSRIs. These classes of medications have been shown to produce ataxia, impair psychomotor function, cause syncope, and lead to additional falls. ${ }^{12}$ Given the results reported here, it would seem that physicians are aware of the potential for benzodiazepines to increase fall risk in elderly patients and are more likely to discontinue their use as compared with other, less commonly prescribed medications listed in the Beers criteria. For example, SSRIs were not included in the 2003 Beers criteria but were newly added to the updated criteria published in 2012. Perhaps repeat data collection could provide further insight into the prescribing appropriateness of this medication class for elderly patients as prescribers become familiar with the updated criteria.

Antipsychotics were the type of PIM most frequently added to patients' regimens on hospital discharge, with antipsychotics newly prescribed during the hospital stay being continued on discharge for 13 patients. Although antipsychotics have many potential uses, such as management of delirium or nausea, sedation, and treatment of various mental health conditions, the balance of risks and benefits must be considered in the decision 
to continue these medications for elderly individuals at risk of recurrent falls.

In 2006, Laroche and others ${ }^{1}$ described the effect of hospital admission on the use of all classes of PIMs (as defined using the Beers criteria) among elderly patients (70 years of age or older) admitted to an acute medical geriatric unit. Lists of all drugs received before admission and at discharge were established by the investigators. Although this study did not focus only on PIMs that influence the risk of falls, the mean number of drugs used by patients decreased during the hospital stay, from $6.2 \pm 3.1$ at admission to $5.4 \pm 2.5$ at discharge $(p<0.001)$. The prevalence of PIM use also decreased, from 66\% (95\% CI 63.8-68.0) on admission to $44 \%$ (95\% CI 41.3-45.9) at discharge. ${ }^{1}$ Although the current study concurred with these results in terms of a statistically significant reduction in PIMs, this change may not be considered clinically significant. The proportion of patients with an overall change in PIMs during the hospital stay may be more clinically relevant.

In 2011, Kragh and others ${ }^{10}$ published a population-based cohort study comparing the use of medications increasing fall risk for older adults 6 months before and 6 months after hip fracture. They found that $67.7 \%$ of study participants had been using medications with potential to increase fall risk before their hip fracture, and $73.4 \%$ were treated with such a drug in the 6 months after the fracture (with opioids excluded from the postfracture analysis, on the assumption that this class of medications was prescribed for pain control).$^{10}$ In particular, the use of sedative or hypnotic medications increased: in the 6 months before the fracture, $36.5 \%$ of study participants were using a sedative or hypnotic medication, compared with 51.7\% at discharge. ${ }^{10}$ Results such as these indicate that clinicians may not always recognize medication use as a modifiable risk factor for falls.

During the follow-up period of the current study, 13 (9\%) of the patients experienced a subsequent fall that resulted in presentation to the emergency department or readmission to hospital. Unfortunately, because of a lower-than-anticipated event rate, the study was underpowered to detect a significant difference in fall recurrence between patients discharged with a reduction in dosage or number of PIMs and those discharged with an increase in dosage or number of PIMs. Detection of a difference in this outcome was limited by the inability to capture recurrent falls that did not result in presentation to hospital or recurrent falls that resulted in presentation or admission to an institution other than the study hospital. Furthermore, the retrospective study design prevented confirmation that medication changes made upon hospital discharge were maintained throughout the follow-up period. This limited the ability to reliably link recurrent falls with PIM changes made on discharge. The specification of a follow-up period based on particular calendar dates (January 1 to December 31, 2012) meant that patients who were enrolled near the end of the study period (i.e., later in 2011) had a shorter follow-up window for the capture of recurrent falls. Future research using a prospective study design with potential for long-term follow-up may be better suited to addressing these limitations and capturing a difference in fall recurrence.

The multivariate analysis showed that admission to the orthopedics service in combination with consultation to the geriatrics service was associated with a reduction in PIMs. The geriatrics consult service is very active at the study hospital, particularly in relation to surgical admitting services, so this finding was not surprising. The connection between these 2 factors was further supported by the observation that admission to the orthopedics service was not associated with de-escalation of PIMs upon univariate analysis but was a significant predictor upon multivariate analysis with geriatric consultation also included in the model. Routine geriatrics consultation for elderly surgical patients has recently been shown to be associated with improved functional recovery, ${ }^{15}$ and the findings of the current study further emphasize this value.

The multivariate analysis showed no association between pharmacist involvement in patient care and a reduction in number or dosage of PIMs on discharge. A pharmacist's involvement in care was identified for only $16 \%$ of the cohort ( 23 patients). Pharmacists are an integral part of the circle of care, and this proportion is likely an underestimate, possibly related to the retrospective nature of the study and the inability to identify pharmacist contributions that were not documented in the medical chart. The pharmacist-patient ratios at the study centre are about 1:40 for the orthopedics service and 1:25 for general medicine. Future studies to evaluate the contribution of pharmacists to reducing the risk of negative outcomes related to polypharmacy should be prospective to allow their interventions to be accurately captured. Retrospective studies may still be of value but must consider the frequency of documentation in calculation of sample sizes.

Admission to hospital could be viewed as an opportunity to reassess the appropriateness of long-term medication use in elderly individuals and to determine whether patients are receiving PIMs or other drug therapy without a recognized indication. Involvement of geriatrics consult services may increase the ability of hospital-based physicians to safely taper or discontinue targeted medications in elderly individuals believed to be at increased risk of harm due to ADEs. Although no significant impact of pharmacist involvement on de-escalation of PIMs was observed in the current study, further research, with better capture of pharmacist interventions, is warranted, as this activity is within the scope of clinical pharmacy practice.

Education for prescribers on the use of published criteria for use of PIMs in elderly individuals is a possible strategy to promote more conservative prescribing and has the potential to improve patient safety. Published criteria do not replace clinical judgment and should be used with consideration of patient- 
specific comorbidities and risk factors. The retrospective nature of the current study prevented evaluation of patient-specific factors that might have contributed to the reduction in PIMs observed in a proportion of the study population. Future prospective studies may be better suited to capture similarities or differences between those who are more likely to experience a reduction in PIMs and those more likely to experience an increase.

Further research on medication use in geriatric patients and the association between PIMs and preventable hospital admissions would be of interest and may promote additional strategies to reduce inappropriate medication use in elderly individuals.

\section{References}

1. Laroche ML, Charmes JP, Nouaille Y, Fourrier A, Merle L. Impact of hospitalization in an acute medical geriatric unit on potentially inappropriate medication use. Drugs Aging. 2006;23(1):49-59.

2. Corsonello A, Pranno L, Garasto S, Fabietti P, Bustacchini S, Lattanzio F. Potentially inappropriate medication in elderly hospitalized patients. Drugs Aging. 2009;26 Suppl 1:31-9.

3. Huang AR, Mallet L, Rochefort CM, Eguale T, Buckeridge DL, Tamblyn R. Medication-related falls in the elderly, causitive factors and preventive strategies. Drugs Aging. 2012;29(5):359-76.

4. Thomas EJ, Brennan TA. Incidence and types of preventable adverse events in elderly patients: population based review of medical records. BMJ. 2000;320(7237):741-4.

5. Freeland KN, Thompson AN, Zhao Y, Leal JE, Mauldin PD, Moran WP. Medication use and associated risk of falling in a geriatric outpatient population. Ann Pharmacother. 2012;46(9):1188-92.

6. Tinetti ME, Baker DI, McAvay G, Claus EB, Garrett P, Gottschalk M, et al. A multifactorial intervention to reduce the risk of falling among elderly people living in the community. N Engl J Med. 1994;331(13):821-7.

7. Hanlon JT, Landerman LR, Fillenbaum GG, Studenski S. Falls in African American and white community-dwelling elderly residents. J Gerontol A Biol Sci Med Sci. 2002;57(7):M473-8.

8. Berdot S, Bertrand M, Dartiques JF, Fourrier A, Tavernier B, Ritchie K, et al. Inappropriate medication use and risk of falls - a prospective study in a large community-dwelling elderly cohort. BMC Geriatr. 2009;9:30.

9. Hartikainen S, Lonnross E, Louhivuori K. Medication as a risk factor for falls: a critical systematic review. J Gerontol A Biol Sci Med Sci. 2007;62(10): 1172-81.
10. Kragh A, Elmstahl S, Atroshi I. Older adults' medication use 6 months before and after hip fracture: a population based cohort study. J Am Geriatr Soc. 2011;59(5):863-8.

11. Egger SS, Bachmann A, Hubmann N, Schlienger RG, Krahenbuhl S. Prevalence of potentially inappropriate medication use in elderly patients: comparison between general medical and geriatric wards. Drugs Aging. 2006;23(10):823-37.

12. American Geriatrics Society 2012 Beers Criteria Update Expert Panel. American Geriatrics Society updated Beers criteria for potentially inappropriate medication use in older adults. J Am Geriatr Soc. 2012;60(4):616-31.

13. Rossat A, Fantino B, Bonque B, Colvez A, Nitenberg C, Annweiler C, et al. Association between benzodiazepines and recurrent falls: a cross-sectional elderly population-based study. J Nutr Health Aging. 2011;15(1):72-7.

14. Leipzig RM, Cumming RG, Tinetti ME. Drugs and falls in older people: a systematic review and meta-analysis. I. Psychotropic drugs. J Am Geriatr Soc. 1999;47(1):30-9.

15. Jones TS, Dunn CL, Wu DS, Cleveland JC, Kile D, Robinson TN. Relationship between asking an older adult about falls and surgical outcomes. JAMA Surg. 2013;148(12):1132-8.

Erin Francis, BSc, BScPharm, ACPR, is with the Pharmacy Department, The Ottawa Hospital-General Campus, Ottawa, Ontario.

Derek Dyks, BScPharm, CGP, is with the Pharmacy Department, The Ottawa Hospital-Civic Campus, Ottawa, Ontario.

Salmaan Kanji, BSc, PharmD, ACPR, is with The Ottawa Hospital Research Institute and the Pharmacy Department, The Ottawa HospitalGeneral Campus, Ottawa, Ontario; the Faculty of Medicine, University of Ottawa, Ottawa, Ontario; and the Faculty of Pharmacy, Université de Montréal, Montréal, Quebec.

Competing interests: None declared.

\section{Address correspondence to:}

Erin Francis

Pharmacy Department

The Ottawa Hospital-General Campus

501 Smyth Road

Ottawa ON K1H 8L6

e-mail: efrancis@toh.on.ca 